\title{
Horizontal transport under wind-induced resonance in stratified waterbodies
}

\author{
Hugo N. Ulloa $\oplus^{*}$ \\ Physics of Aquatic Systems Laboratory, Margaretha Kamprad Chair, École Polytechnique Fédérale de \\ Lausanne, CH-1015 Lausanne, Switzerland
}

George Constantinescu 10

Civil and Environmental Engineering, IIHR-Hydroscience and Engineering, The University of Iowa, Iowa City, Iowa 52242, USA

Kyoungsik Chang $\odot$

School of Mechanical Engineering, University of Ulsan, Ulsan 44610, South Korea

\begin{abstract}
Daniel Horna-Munoz 10
Environmental Engineering Department, Centro de Investigación y Tecnología del Agua (CITA), Universidad de Ingeniería y Tecnología (UTEC), Barranco, Lima 15063, Peru

Océane Hames

Physics of Aquatic Systems Laboratory, Margaretha Kamprad Chair, École Polytechnique Fédérale de Lausanne, CH-1015 Lausanne, Switzerland

Alfred Wüest 10

Physics of Aquatic Systems Laboratory, Margaretha Kamprad Chair, École Polytechnique Fédérale de Lausanne, CH-1015 Lausanne, Switzerland

and Eawag, Swiss Federal Institute of Aquatic Science and Technology, Surface Waters-Research and Management, CH-6047 Kastanienbaum, Switzerland
\end{abstract}

(Received 6 September 2019; accepted 27 April 2020; published 20 May 2020)

Periodic winds acting on a stratified waterbody can amplify normal modes of motion and enhance the basin-scale circulation via resonance. Here, we use idealized largeeddy simulations to investigate the flow features and quantify the horizontal transport in periodically wind-forced stratified basins. Motivated by observations in lakes, we focus on systems in which daily winds either resonate with the second vertical basin-scale internal mode, V2H1 (case 1), or the first vertical basin-scale internal mode, V1H1 (case 2). In particular, we analyze the case when strong nonlinearities affect the evolution of the V2H1 mode (case 3). To achieve these three resonance scenarios, we hold the basin morphology and the periodic forcing invariant, but change the background stratification. Our results show that a quasilinear $\mathrm{V} 2 \mathrm{H} 1$ modal response has more active mass transport in the boundary regions than does the quasilinear $\mathrm{V} 1 \mathrm{H} 1$ case. This difference arises from the lack of midlayer horizontal transport in the V1H1 mode, whereas the midlayer current in the V2H1 mode intensifies the transport along the slopes in both directions by splitting the flow into two branches, one running upslope and one running downslope. Nonlinear dynamics further amplify the along-slope transport in case 3, in which a second mode, an undular borelike wave, emerges from the periodic forcing. This study shows that the horizontal transport under wind-induced resonance is sensitive to the amplified mode of

\footnotetext{
*Corresponding author: hugo.ulloa@epfl.ch
} 
motion in the stratified basin and that nonlinear flow dynamics can considerably enhance mass transport in sloping regions.

DOI: 10.1103/PhysRevFluids.5.054503

\section{INTRODUCTION}

Horizontal transport regulates the heat and matter distribution in aquatic systems, such as lakes [1-4]. In stratified waterbodies, periodic winds induce surface and internal flows and can amplify normal modes via resonance [1]. As a result, an oscillation mode with a frequency close to the forcing frequency [5-8] is overamplified relative to the rest of the modes that the system admits. Observations in lakes show that basin-scale internal waves, commonly called internal seiches [9], are susceptible to resonating with diurnal winds [1,7,10-16]. Examples of these modes of motions are the first vertical, first horizontal mode, V1H1 [Fig. 1(a)] [1,7,10,12,17], and the second vertical, first horizontal mode, V2H1 [Fig. 1(b)] [11,16]. Although any normal mode with a natural frequency near the forcing frequency can be theoretically amplified by resonance, higher vertical mode seiches (e.g., V3H1, V4H1) are more difficult to identify in the field [13,14] than V1H1 and V2H1. These latter modes usually have strong signatures both in the horizontal currents and the isotherms displacement [18-20].

Wind-induced resonance is a particularly interesting hydrodynamic regime since the entire basin is driven into motion. In this scenario, the amplified basin-scale circulation can physically connect ecologically relevant regions, such as nearshore and interior waters [21]; help to ventilate deep waters [22,23]; resuspend sediment and nutrients [24,25]; and, generally enhance lateral exchange $[15,26]$. While the cross-shore mass transport between nearshore sloping boundaries and interior waters due to shoaling internal waves has been investigated [27-30], the impact of resonance on basin-scale flows has been little explored and quantified. How does the horizontal transport change over the resonant cycle and the distance from the lateral boundaries? Is the horizontal transport affected by the amplified modal response hosted by a system? Addressing these questions requires finding systems under resonant regimes and determining their spatiotemporal mass and flow distribution. Here, we investigate this problem via a series of idealized numerical experiments with dimensions of a real system.

Inspired by observations in stratified lakes and the challenge of measuring the horizontal transport in such aquatic systems, we quantify the horizontal mass transport in periodically windforced, stratified waterbodies under resonance. We examine systems whose amplified basin-scale internal modes are the commonly observed V2H1 (case 1) and V1H1 (case 2). In this study, cases 1 and 2 are characterized by quasilinear (QL) wave dynamics and are denoted as QL-V2H1 and QL-V1H1, respectively [Fig. 1(e)]. These cases are used to provide insights into how the horizontal transport is affected by changes in the vertical modal structure. Additionally, we examine a third case in which the V2H1 internal wave is affected by strong nonlinearities (NL) that resemble previous observations [e.g., 10,31]. We denote this case as NL-V2H1 [Fig. 1(e)]. Ulloa et al. [20] investigated the hydrodynamics of QL-V2H1 to delve into the macroscopic dynamics of Lake Alpnach (Switzerland). QL-V2H1 shows good agreement with the observations of Lake Alpnach, including the bottom boundary layer currents and the vertical distribution of the kinetic energy dissipation rate. Here, our numerical results show how the hydrodynamics and transport may dramatically change due to varying the background stratification in the waterbody (e.g., density difference between the top and bottom constant-density layers and the thickness of the middle layer) and the basin-scale internal wave dynamics.

In Sec. II, we present the method adopted to perform and analyze the numerical experiments. Section III shows the basin-scale and local features of the horizontal transport. Then, we assess the impact of the horizontal transport on the rate of mass exchange and the flushing timescales of nearshore waters. Finally, we discuss our results in Sec. IV and summarize our findings in Sec. V. 

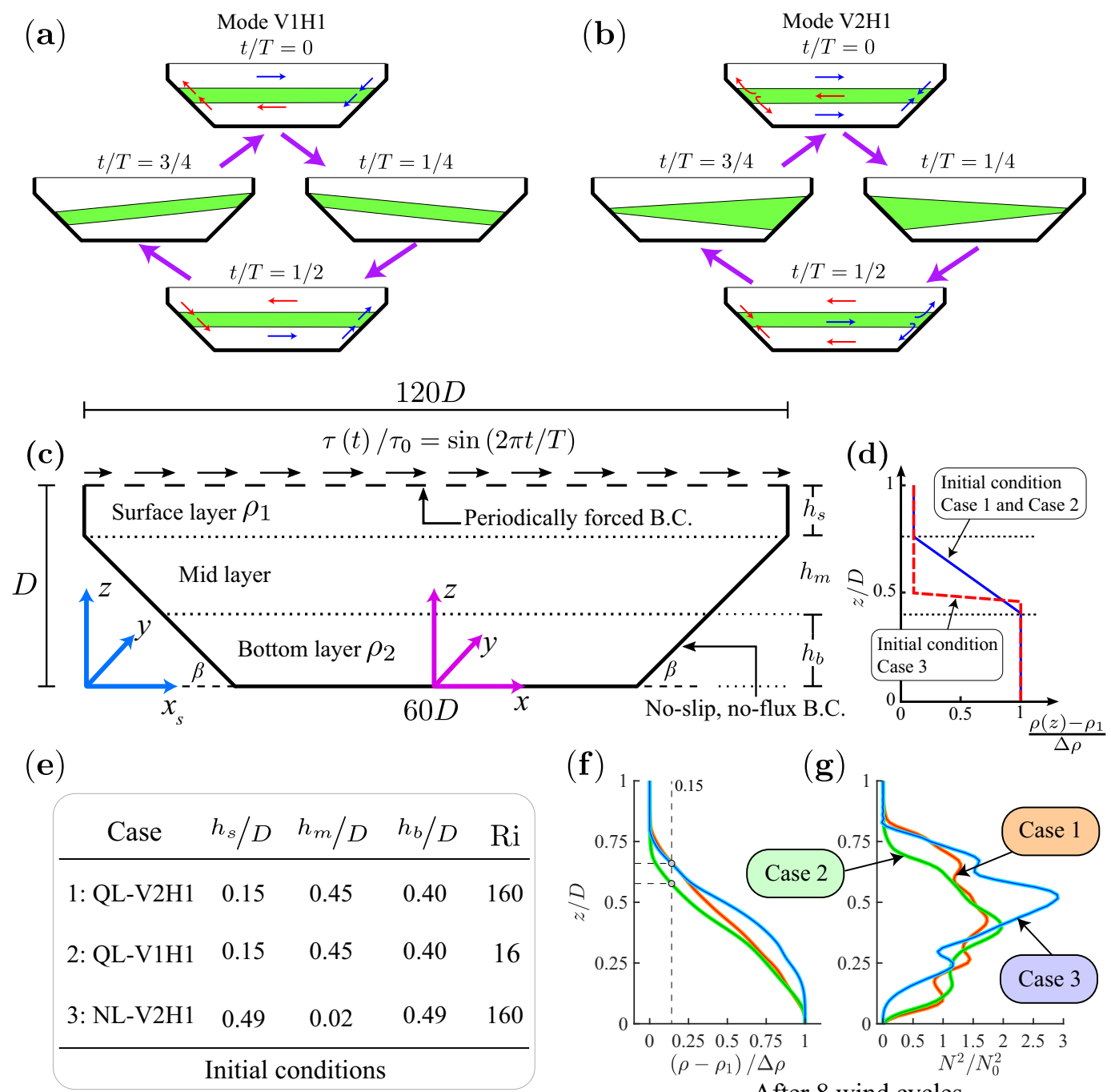

\begin{tabular}{ccccc} 
Case & $h_{s} / D$ & $h_{m} / D$ & $h_{b} / D$ & $\mathrm{Ri}$ \\
\hline 1: QL-V2H1 & 0.15 & 0.45 & 0.40 & 160 \\
2: QL-V1H1 & 0.15 & 0.45 & 0.40 & 16 \\
3: NL-V2H1 & 0.49 & 0.02 & 0.49 & 160 \\
\hline \multicolumn{5}{c}{ Initial conditions }
\end{tabular}
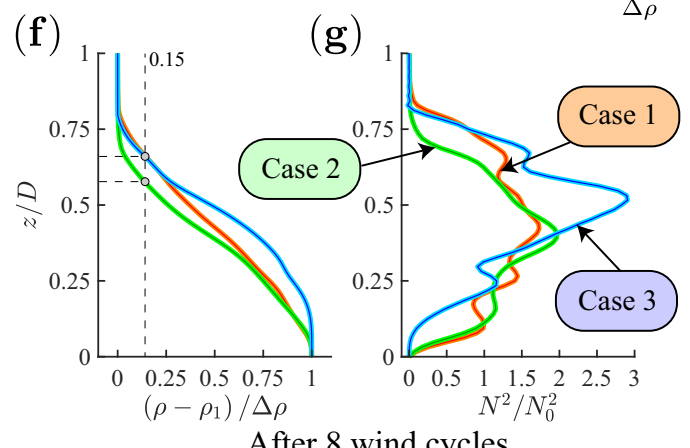

After 8 wind cycles

FIG. 1. (a),(b) Schematics of modes V1H1 and V2H1, respectively. (c) Sketch of the computational domain of a periodically wind-forced stratified basins. $D$ is the maximum depth. $h_{s}, h_{m}$, and $h_{b}$ are the surface-, mid-, and bottom-layer thicknesses, respectively. $s \equiv \tan (\beta)$ is the slope of the side bottom. $\rho_{1}$ and $\rho_{2}$ are the initial surface- and bottom-layer densities. $\tau(t)$ is a sinusoidal time-varying shear stress acting at the free surface with period $T$ and amplitude $\tau_{0}$. Two reference systems are used in this work: one located at the center bottom of the basins, $(x, y, z)$ in magenta color, and one at the left bottom corner of the numerical domain, $\left(x_{s}, y, z\right)$ in cyan color. (d) Initial density profile. The blue line corresponds to cases 1 and 2, while the red dashed line corresponds to case 3. (e) Summary of the layer thicknesses for each case along with their Richardson number. Nondimensional vertical profiles of the (f) background density, $\left(\rho-\rho_{1}\right) / \Delta \rho$, and (g) fluid stability, $N^{2} / N_{0}^{2}$, after eight wind cycles. $N_{0}^{2}$ is the initial fluid stability of the stratified midlayer.

\section{METHOD}

\section{A. Numerical method}

The governing equations, the large-eddy simulation (LES) model, and the numerical method are described in detail in Ulloa et al. [20]. Here, we briefly review the main features of the model. 
The Boussinesq approximation is employed to account for stratification effects. The Navier-Stokes and density transport equations are solved in their nondimensional form. The length scale $D$ is the depth of the basin away from the side slopes, while the velocity scale $U_{0}$ is selected to be near to the peak velocity magnitude inside the basin due to wind-induced flow at the free surface. The nondimensional density is defined as $\tilde{\rho}=\left(\rho-\rho_{1}\right) / \Delta \rho$, where $\rho$ is the dimensional density and $\Delta \rho=\rho_{2}-\rho_{1}$ is the difference between the maximum (bottom-layer) density $\rho_{2}$ and the minimum (top-layer) density $\rho_{1}$, respectively, at the start of the simulation. The three parameters in the nondimensional governing equations are the Reynolds number, $\operatorname{Re} \equiv U_{0} D / \nu$, the Richardson number, $\mathrm{Ri} \equiv g\left(\Delta \rho / \rho_{1}\right) D / U_{0}^{2}$, and the molecular Prandtl number, Pr $\equiv v / \kappa$, where $v$ is the molecular viscosity, $\kappa$ is the molecular diffusivity, and $g$ is the gravitational acceleration. The subgrid-scale viscosity $\nu_{\mathrm{SGS}}$ and subgrid-scale diffusivity $\kappa_{\mathrm{SGS}}$ are estimated from the instantaneous velocity and density fields using the dynamic Smagorinsky model [32]. We limit our analysis to stratified waterbodies in which the Earth's rotation plays a negligible role in the basin-scale internal wave dynamics (see discussion in Ulloa et al. [20]).

In the present finite-volume approach $[33,34]$, the conservative forms of the Navier-Stokes equations are integrated in time using a semi-implicit iterative method and are discretized in space using a second-order central scheme. This minimizes the numerical dissipation and damping of the resolved eddies in the simulations, which is critical for accurate results using LES. The pressure Poisson equation is solved using a multigrid approach. The numerical algorithm is second-order accurate in both space and time and discretely conserves energy [35]. A conserved scalar equation is solved for the nondimensional density. The advective term in this equation is discretized using the quadratic upstream interpolation for convective kinematics (QUICK) scheme. Directly relevant for the flows investigated in the present study are three-dimensional (3D) numerical studies of intrusion currents [36], gravity currents propagating over inclined surfaces, or interacting with them [37,38], studies of internal bore and internal wave dynamics [34,39], and studies of bioconvection in lakes [40] conducted using the same flow solver. These investigations validate the numerical predictions using data from laboratory experiments and provide a comparison with shallow water theory results where available.

\section{B. Simulations setup}

Simulations are performed in a quasi-trapezoidal-shaped basin of depth $D=40 \mathrm{~m}$ in the $z$ axis, a free surface of length $120 D$, and a bottom horizontal length of $60 D$ in the streamwise $x$ axis [Fig. 1(a)]. The domain in the $x$ axis is bounded by vertical walls of depth $0.15 D$. The slope of the inclined lateral boundaries is $s \approx 3 \%$. The domain width along the spanwise $y$ axis is $1.6 D$, which is essential to capture vortex stretching phenomena and the development of 3D turbulence. These dimensions approximate a streamwise-vertical cross section of Lake Alpnach, Switzerland [11] along the direction of the dominant wind, and the flow is assumed to be homogeneous in the $y$ direction.

The horizontal and lateral inclined boundaries of the basin are treated as no-slip boundaries. At the top, a sinusoidal shear stress boundary condition is imposed: $\tau_{x}=\tau \equiv \tau_{0} \sin (2 \pi t / T)$ and $\tau_{y}=0$, where $t$ is the time, $T=24 \mathrm{~h}$ is the wind period, and $\tau_{0}=C_{D} \rho_{\text {air }} U_{10}^{2}=0.025 \mathrm{~N} \mathrm{~m}^{-2}$ [4] is the maximum value of the wind-induced shear stress. The magnitude of $\tau_{0}$ corresponds to winds with a speed of about $4 \mathrm{~m} \mathrm{~s}^{-1}$ and a wind-induced velocity scale of $U_{0}=0.05 \mathrm{~m} \mathrm{~s}^{-1}$ [11,18]. As the top surface is assumed to be a rigid lid, the vertical velocity is set equal to zero. The density gradient at the top, lateral, and bottom boundaries is also set to zero. As the flow is assumed to be homogeneous in the $y$ direction, periodic boundary conditions are imposed for all variables at the spanwise boundaries.

The parametrization of the three study cases is presented next. A stable three-layer initial distribution for the density is assumed for all cases. The initial vertical density structures are chosen to obtain (theoretical) $24 \mathrm{~h}$ periods for the considered basin-scale internal modes. The density is uniform in the top (epilimnion) layer $\left(\rho=\rho_{1}\right)$ of thickness $h_{s}$ and in the bottom (hypolimnion) layer 
( $\rho=\rho_{2}=999 \mathrm{~kg} \mathrm{~m}^{-3}$ ) of thickness $h_{b}$. The maximum density differences are $\Delta \rho=1 \mathrm{~kg} \mathrm{~m}^{-3}$ for cases 1 and 3 and $\Delta \rho=0.1 \mathrm{~kg} \mathrm{~m}^{-3}$ for case 2 . A midlayer of thickness $h_{m}$ is characterized by a linear density distribution. Figure 1(b) shows the initial vertical profiles of the nondimensional density $\tilde{\rho}$. At $t /\left(D / U_{0}\right)=0$, the velocity field is set equal to zero in the middle and bottom layers, while random (uniformly distributed) values with a mean of $0.03 U_{0}$ are assigned to the three velocity components inside the top layer. This is done so that turbulence develops faster inside the basin once the wind forcing becomes active. The heights of the three layers are identical in cases 1 and $2\left(h_{s}=0.15 D, h_{m}=0.45 D, h_{b}=0.4 D\right)$. Case 3 is close to a two-layer $\left(h_{m}=h_{s}=0.49 D\right)$ initial density distribution with a sharp interface, as the height of the midlayer is only $h_{m}=0.02 D$. Given that the density is assumed to be a function only of the temperature, the molecular Prandtl number is assumed to be equal to 6 . The Richardson number, $\mathrm{Ri}$, is set equal to $\mathrm{Ri}=160$ in cases 1 and 3 . This corresponds to an initial temperature difference between the top and bottom layers of about $9{ }^{\circ} \mathrm{C}$. In case $2, \mathrm{Ri}=16$, which corresponds to a reduced initial temperature difference of only $0.9{ }^{\circ} \mathrm{C}$. Besides the value of the Richardson number, all the other flow variables and initial conditions are identical in cases 1 and 2.

All three simulated cases have identical meshes. The flow is resolved in the homogeneous direction $y$ using 64 nodes. Nearly 300000 grid points are used to mesh the flow domain in a streamwise-vertical cross section $(x, z)$. Away from the free surface and the no-slip boundaries, the cell size is close to $0.025 \mathrm{D}$ in all three directions. The grid resolution is refined to about $0.001 D$ near the free surface and the flat bottom boundary to resolve the wind-induced and bottom boundary layers. The nondimensional time step is $0.002\left(D / U_{0}\right)$. To properly resolve the flow and temperature fields in this numerical setting, we integrated the nondimensional equations of motion with an effective Reynolds number of $\operatorname{Re}=10^{5}$ [20], which is one order of magnitude lower than its real-scale magnitude. The code was run on 128 processors on a high-performance computing (HPC) cluster.

Here, we do not address the problem of how a basin-scale internal mode enters into or out of resonance $[10,12]$. Instead, we detail how the resonance regimes are achieved. The periodic solutions for the three cases are analyzed after the turbulent flow has become quasiperiodic. Based on an inspection of the $2-D$ spanwise-averaged density and velocity flow fields and of the time series of the density and velocity at several points, the flow can be considered quasiperiodic for $t / T>7$. Thus, the flow hydrodynamics is analyzed in all cases for $9<t / T<10$ (tenth cycle). For simplicity, we consider that $t / T=9.0$ is $t / T=0$ at the start of the tenth wind (diurnal) cycle analyzed in this work.

As the flow becomes quasiperiodic, the vertical density distribution becomes smoother, as illustrated in Fig. 1(d). Due to the periodic shearing and the mixing at mid depths, the three cases show an increase in the midlayer thickness. Case 3 shows evidence of the largest difference compared to its initial midlayer thickness $\left(h_{m}=0.02 D\right)$. Although cases 1 and 2 start from the same density distribution, case 2 develops a thicker top layer $(\sim 0.3 D)$ than case $1(\sim 0.2 D)$. Additionally, in case 1, the density distribution beneath the top layer is almost linear, with an intense smoothing of the bottom layer. Figure 1(e) illustrates, for each case, the nondimensional background profile of the square of the Brunt-Väisälä frequency, $N^{2}$. Case 3 has its maximum peak at $z / D=0.51$, whereas cases 1 and 2 have their maximum peaks at $z / D=0.42$ and $z / D=0.40$, respectively. It should also be noted that the background density distributions have evolved in such a way that their respective $N^{2}$ profiles show more than one local peak. This feature is especially visible for cases 1 and 3 .

\section{RESULTS}

We first present a global characterization of the basin-scale internal motions amplified by periodic diurnal winds. Then, we take a closer look at the along-slope and near-bottom mass transport. Finally, we map the horizontal mass exchange and the flushing timescale of nearshore waters as a function of space and time. 

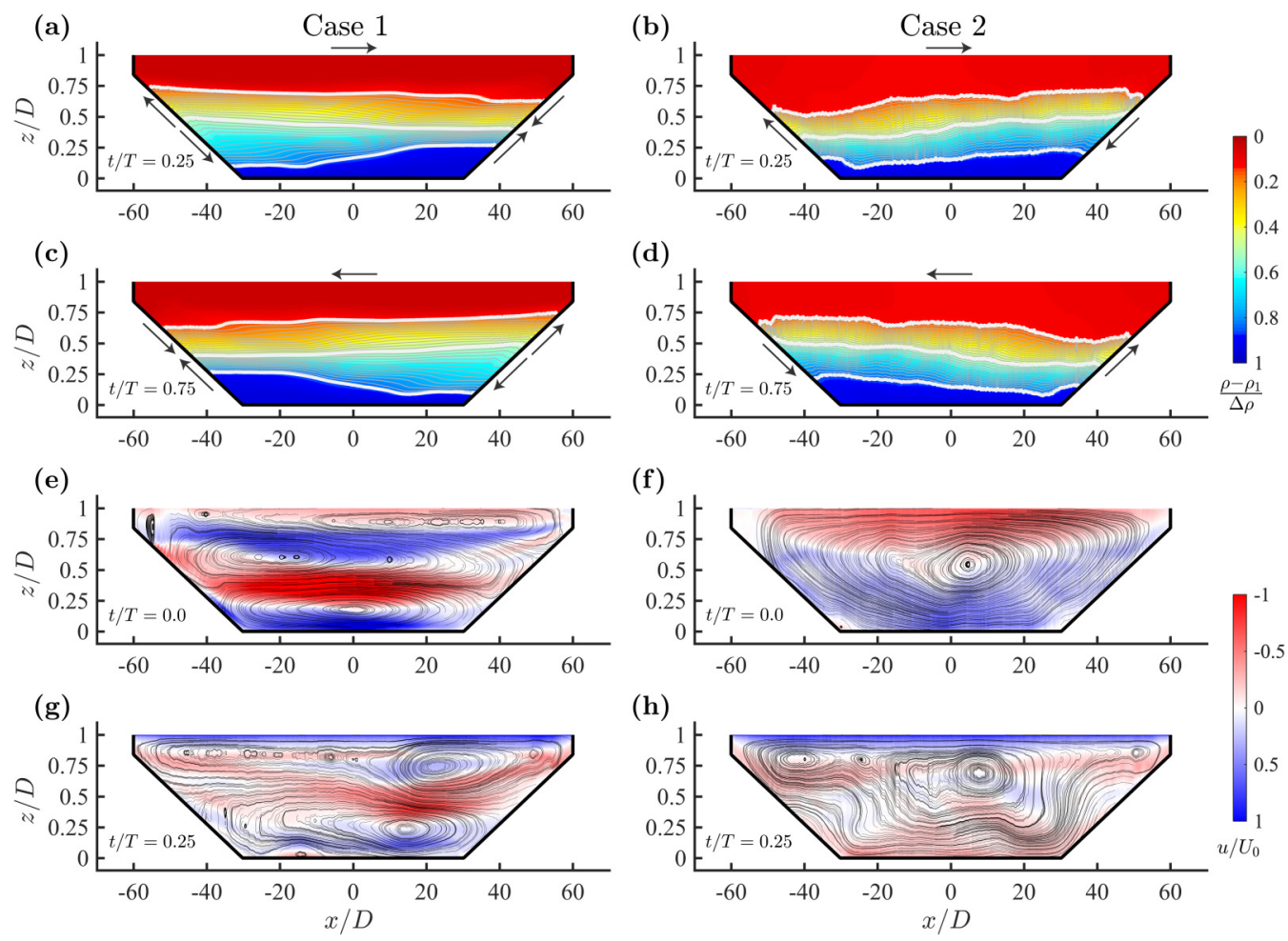

FIG. 2. Wind-forced, basin-scale response of the (a)-(d) spanwise-averaged density and (e)-(h) streamwise flow, $u$. Fields are shown in nondimensional form. Case 1: (a) and (c) show the density (along isopycnals in the midlayer tracking its boundaries and mean density contour) characterized by the second vertical, first horizontal (V2H1) modal response (three layers). Case 2: (b) and (d) show the same fields, but the fluid response is characterized by the first vertical, first horizontal (V1H1) modal response. Both modes of motion have a period near $T$. Arrows in (a)-(d) show flow direction. Flow circulation in (e) and (f) are shown with a time $t / T=0.0$ to illustrate the overall basin-scale flow structure. (g), (h) The flow circulation at a time $t / T=0.25$.

\section{A. Basin-scale internal motions under resonance}

\section{Quasilinear (QL) V2H1 and V1H1 modes of motion}

Figure 2 shows the modal response for cases 1 and 2. Linear normal modes can well characterize their basin-scale features [41,42]. Nevertheless, they are not free of nonlinearities. Resonance magnifies the amplitude of the internal seiches. In both cases, we observe the formation of weak fronts attributed to nonlinear steepening [see white isopycnals in Figs. 2(a) and 2(b)], albeit not strong enough to lead to dispersive processes [43-46]. Here, we denote their basin-scale response as quasilinear $(\mathrm{QL})$ modes of motion.

In case 1 , the oscillatory wind amplifies the QL-V2H1 mode, which is characterized by threelayer dynamics. Figures 2(a) and 2(c) illustrate the QL-V2H1 density response at $t / T=0.25$ and 0.75 . At these wind phases, the maximum stretching and squeezing of the midlayer isopycnals occur over the sloping boundaries [11]. In addition, Figs. 2(e) and 2(g) show the flow circulation for the same wind phases. The flow circulation is characterized by two basin-scale cells that occupy most of the stratified basin, including the shallow nearshore regions. When there is wind shear at the surface, a thin surface layer flows parallel to the wind direction, whereas a mid-depth layer flows against the wind, from nearshore toward the interior. Interestingly, once the wind diminishes and the fluid is free to oscillate by inertia, there is a significant enhancement of horizontal currents, specifically at 

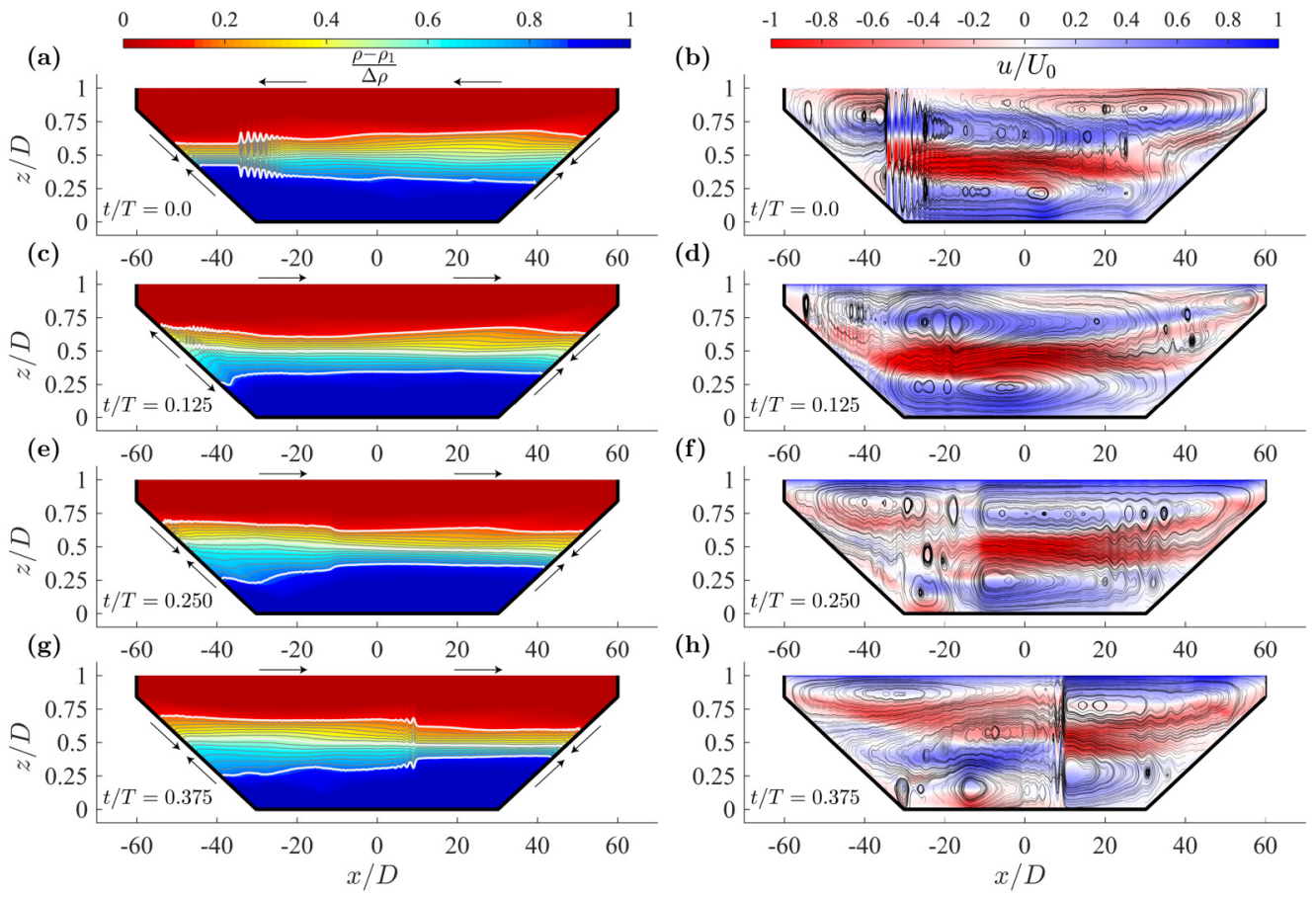

FIG. 3. Wind-forced, basin-scale, nonlinear response of case 3. (a),(c),(e),(g) The spanwise-averaged density along with isopycnals that highlight the vertical displacement of the midlayer. (b),(d),(f),(h) The spanwise-averaged streamwise velocity along with streamlines. Fields are shown in nondimensional form.

mid-depth and deep water. These currents generate convergence and divergence of fluid along the slopes.

In case 2, the oscillatory wind amplifies the QL-V1H1 mode. This mode of motion is characterized by two-layer dynamics, with a pycnocline rocking up and down along the side slopes [Figs. 2(b) and 2(d)]. A basin-scale circulation cell takes place over most of the system; however, it barely involves the shallow regions [Fig. 2(f)]. Similar to case 1, the wind forces a thin surface current in the same direction, while deeper waters flow in the opposite direction following tortuous paths [Fig. 2(h)]. In case 2, the free flow evolution also leads to stronger currents than the ones under forced conditions. Unlike case 1, however, horizontal transport is suppressed at mid depths, so the horizontal transport is concentrated within the upper and deeper layers.

\section{Nonlinear (NL) V2H1 mode of motion}

The flow dynamics of case 3 vary strikingly compared to the previous cases. Figure 3 illustrates the density and flow response during the first half of the diurnal phase $(0.0 \leqslant t / T \leqslant 0.5)$; the second half is fully antisymmetric. The basin-scale internal wave is characterized by a second-mode undular bore [47] [Figs. 3(a), 3(e), 3(g)]; its propagation shows a sharp flow discontinuity at the wavefront [Figs. 3(b), 3(d), 3(f), 3(h)]. This steep front-resembling a shock wave-is followed by a train of second-mode solitary-type waves. The macroscopic structure of this nonlinear (NL) basin-scale internal wave, NL-V2H1, is similar to the internal surge observed in Loch Ness by Thorpe [10,48], but characterized by the second mode.

Even though cases 1 and 3 exhibit similar global fluid patterns, their flow propagations reveal remarkable contrasts. Unlike the QL-V2H1 response in case 1, the wavefront of the NL-V2H1 in case 3 divides the fluid and flow into two different regions: the one downstream of the wave, in 


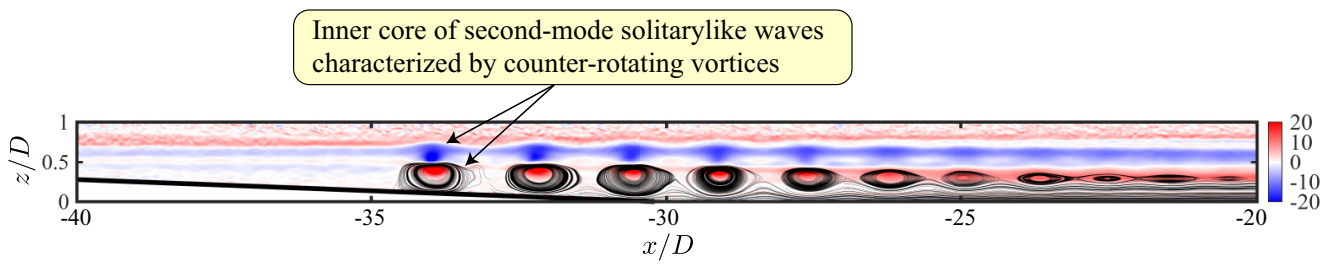

FIG. 4. Case 3: Close-up of the inner core circulation induced by the nonlinear internal gravity wave resulting from the periodic wind forcing. Nondimensional vorticity, $\omega_{y} /\left(U_{0} / D\right)$, along with streamline in the plane $x-z$ at $t / T=9.0$. The aspect ratio of the figure is $2: 1$. Streamlines are not shown over the upper inner core circulation to highlight the vorticity patches.

which the fluid remains almost quiescent, and the one upstream of the wave, in which the fluid is displaced from its equilibrium position by the undular bore propagation [Figs. 3(a) and 3(g)]. Figures $3(\mathrm{e})-3(\mathrm{~h})$ highlight this feature in the streamwise velocity. The horizontal transport stems from a vigorous lateral current in the midlayer, bounded vertically by weaker currents flowing in the opposite direction. In contrast to the QL-V2H1 dynamics in case 1, the impact of the internal bore on the sloping bottom causes a weak stretching of the isopycnals, while their compression is negligible on the opposite slope [Figs. 3(c) and 4(b)].

Figure 4 shows a close-up of the vorticity field of the solitarylike waves developed from the NL$\mathrm{V} 2 \mathrm{H} 1$ in case 3 . Each of them has an inner circulation pattern characterized by two quasisymmetric cells with opposite vorticity signs. This localized inner circulation has been observed previously within second-mode internal solitarylike waves propagating [as in Figs. 3(a) and 3(b)] in a still fluid environment [e.g., 49-51]. Here, however, we observe the case of an internal undular bore propagating with and against the wind-induced horizontal currents, as shown in Figs. 3(g) and 3(h). When the bore propagates against the wind-induced current [e.g., Fig. 3(f)], the results show a drop in the midlayer velocity magnitude. This phenomenon occurs in enclosed basins when the winddriven flow velocity and the wave phase speed are mismatched. The linear wave frequency and phase speed of the excited basin-scale internal mode can be obtained by resolving the Sturm-Liouville eigenvalue problem [41,42]. However, this mode is amplified by resonance and its properties are affected by nonlinearities.

\section{Propagation speed of basin-scale motions}

To obtain the effective phase speed of the dominant modes of motion, we examine the isopycnal displacement as a function of space and time to compute the characteristics of these modes. Figure 5 shows Hovmöller diagrams for the nondimensional vertical displacement of an isopycnal, $\langle\eta\rangle_{\mathrm{sw}} / D$, near the pycnocline, $\tilde{\rho}=0.15$, on the $x / D-t / T$ plane. The time vertical axes in Figs. 5(a) $-5(\mathrm{c})$ and the time horizontal axis in Fig. 5(d) are expressed in terms of the wind phase $(0 \leqslant t / T \leqslant 1)$.

For case 1, the wave-front position in the $x / D-t / T$ plane allows an estimate of the average phase speed of the QL-V2H1 seiche, $\bar{c}_{1}$ [red dashed lines in Fig. 5(a)]. For case 2, however, the QLV1H1 seiche shows no front that can be used to track its position. Its average phase speed, $\bar{c}_{2}$, is instead obtained by computing the time required to reach the maximum and minimum vertical displacements at the sloping lateral boundaries [red dashed lines in Fig. 5(b)]. By contrast, the wave front in case 3 has a well-defined position on the $x / D-t / T$ plane [red dashed lines in Fig. 5(c)]. The average phase speed of the NL-V2H1 wave, $\bar{c}_{3} / U_{0}=1.90$, is higher than that of QL-V1H1, $\bar{c}_{2} / U_{0}=1.49$, and lower than that of QL-V2H1, $\bar{c}_{1} / U_{0}=2.01$.

Considering the estimated magnitudes of $\bar{c}_{1}$ and $\bar{c}_{3}$, and the horizontal length along which the waves propagate, we extrapolate the evolution of their wave fronts forward in time. We find that the QL-V2H1 seiche has the period of wind forcing, $T$, whereas the NL-V2H1 wave has a slightly longer period, close to $1.05 \mathrm{~T}$. The latter leads to a diurnal delay on the NL-V2H1 phase relative to the wind-induced circulation [Fig. 5(d)]. This implies that the NL-V2H1 wave has the same 

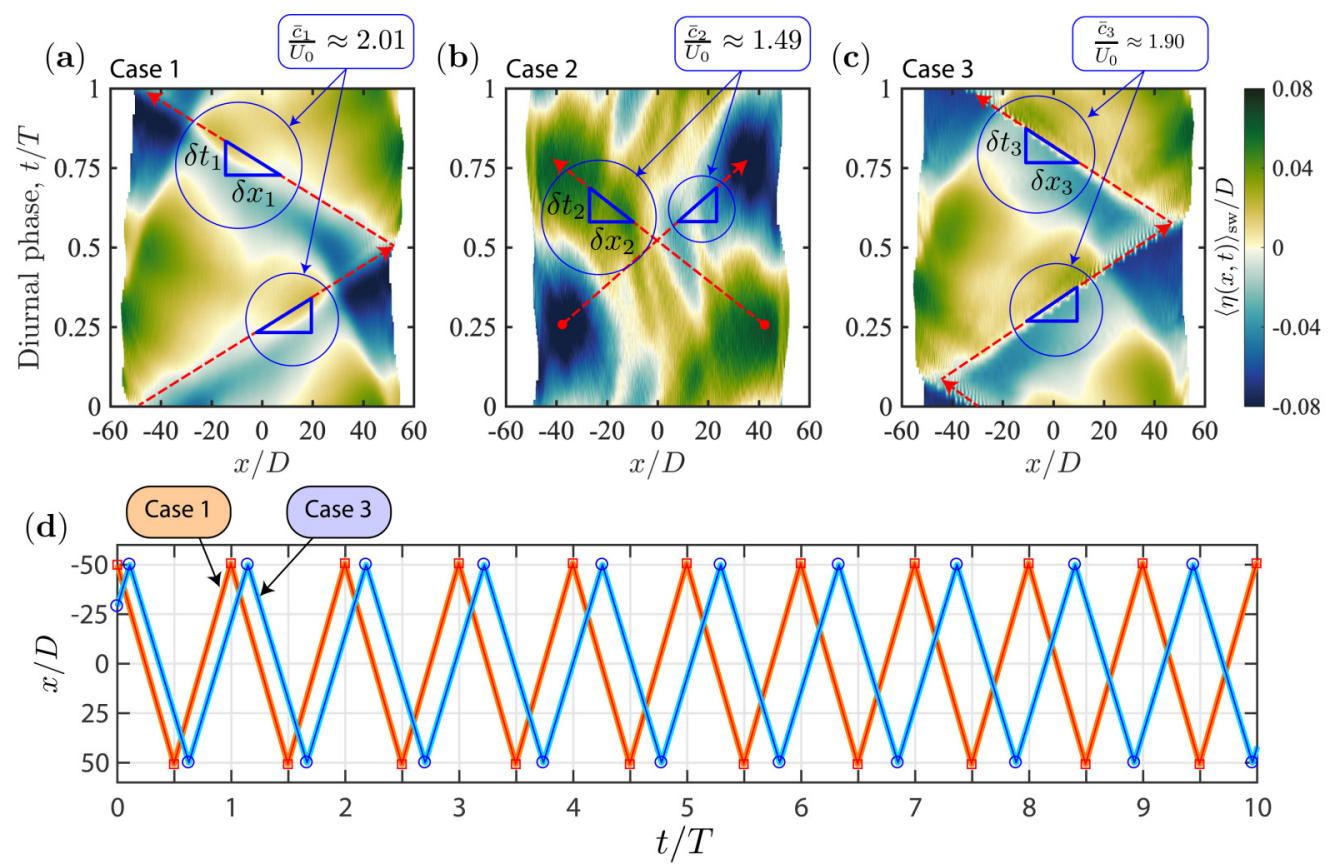

FIG. 5. Hovmöller diagram for basin-scale wave propagation. (a)-(c) The spanwise-averaged isopycnal displacement, $\langle\eta\rangle_{\mathrm{sw}} / D$, as a function of $x / D$ and $t / T$ for $\left(\rho-\rho_{1}\right) / \Delta \rho=0.15$ (see top bold white lines in Figs. 2 and 3). The dashed red lines denote the characteristic curves of the basin-scale internal waves along with their average phase speed (blue triangles). The red dashed lines denote the characteristic curves in the $x / D-t / T$ plane. The average slope of these curves provides an estimation of the basin-scale internal wave phase speed shown in the upper boxes for each case. (d) The extrapolated position of the wave front for cases 1 and 3 as a function $x / D$ and $t / T$.

relative phase as the diurnal wind (Fig. 3) every 20 oscillatory cycles. As a consequence of the timedependent diurnal shift between the wind phase and the NL-V2H1 wave phase, the spatiotemporal distribution of the horizontal transport can either be amplified or weakened over the 20 wind cycles.

\section{B. Along-slope and near-bottom processes}

\section{Shoaling process}

We examine the interactions between the basin-scale internal seiches and the sloping boundaries, focusing on the shoaling process of the NL-V2H1 wave. The train of solitarylike waves developed in case 3 is $\sim 15 D$ long, and its leading wave amplitude approaches $A_{0} \approx 0.1 D$ [Fig. 6(a)] before reaching the sloping boundary. The leading second-mode solitarylike wave in case 3 has an Iribarren number $[44,52,53] \mathrm{I}_{\mathrm{r}} \equiv s / \sqrt{A_{0} / \lambda} \approx 0.12$. Its shoaling behavior is illustrated in Figs. 6(c), 6(f), 6(i), and 6(1). As the train of waves approaches the sloping boundary, the presence of the bottom modifies the lower half of the second-mode waves, so that they diminish in amplitude and speed relative to the upper side. The latter affects the wave symmetry with respect to the pycnocline [Fig. 6(f)]. When the leading solitarylike wave starts colliding with the sloping boundary, its second-mode structure degenerates into mode-one waves of elevation that propagate upslope [Fig. 6(i)]. A similar shoaling process for second-mode solitary waves has been recently reported in laboratory and numerical experiments by Carr et al. [31], who found that the wave disintegration resembles the fission process characterized by Aghsaee et al. [52] and Arthur and Fringer [54]. In our experiments, however, the 


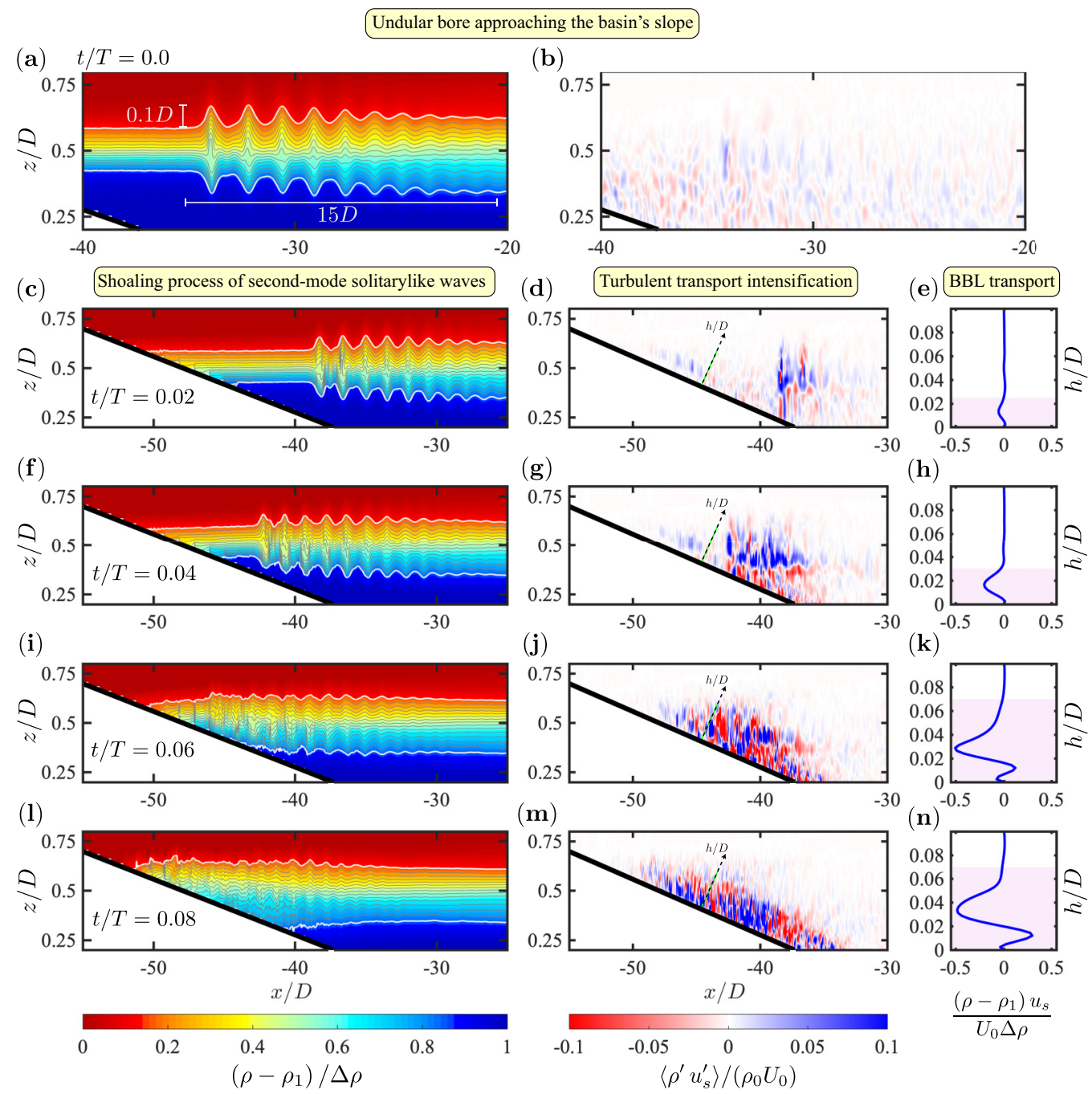

FIG. 6. Case 3. Undular borelike internal wave resulting from the periodic wind forcing. (a) Close-up to the density distribution of the solitarylike internal waves approaching the sloping boundary along with (b) the horizontal turbulent transport at $t / T=0.0$ and $y / D=0.5$. (c),(f),(i),(l) Close-up of the undular internal bore interacting with the sloping bottom along with the respective $(\mathrm{d}),(\mathrm{g}),(\mathrm{j}),(\mathrm{m})$ horizontal turbulent transport and $(\mathrm{e}),(\mathrm{h}),(\mathrm{k}),(\mathrm{n})$ along-slope BBL transport. Fields are shown in nondimensional form and the time interval between the panels is $\Delta T / T=0.02$.

shoaling processes occur periodically. By contrast, the seiches QL-V2H1 (case 1) and QL-V1H1 (case 2) have a wave-slope interaction described by a surging breaker (not shown).

Figures 6(d), 6(g), 6(j), 6(m) and Figs. 6(e), 6(h), 6(k), 6(n), respectively, show the along-slope turbulent transport, $\left\langle\rho^{\prime} u_{s}^{\prime}\right\rangle$, and the bottom boundary layer transport (BBLT), $\left(\rho-\rho_{1}\right) u_{s}$, during the shoaling process. The BBLT is measured at $x / D=-45$, across the normal axis $h / D$ shown in Figs. 6(e), 6(h), 6(k), and 6(n). A strengthening of the transport occurs as the lower half of the waves interacts with the sloping boundary, generating intensive shear and small-scale fluctuations around $h / D \approx 0.015$. As the solitarylike waves degenerate and propagate upward, the transport is confined and intensified near the bottom. 
(a) $\quad(x, z)_{\star} / D=(-52.5,0.61)$
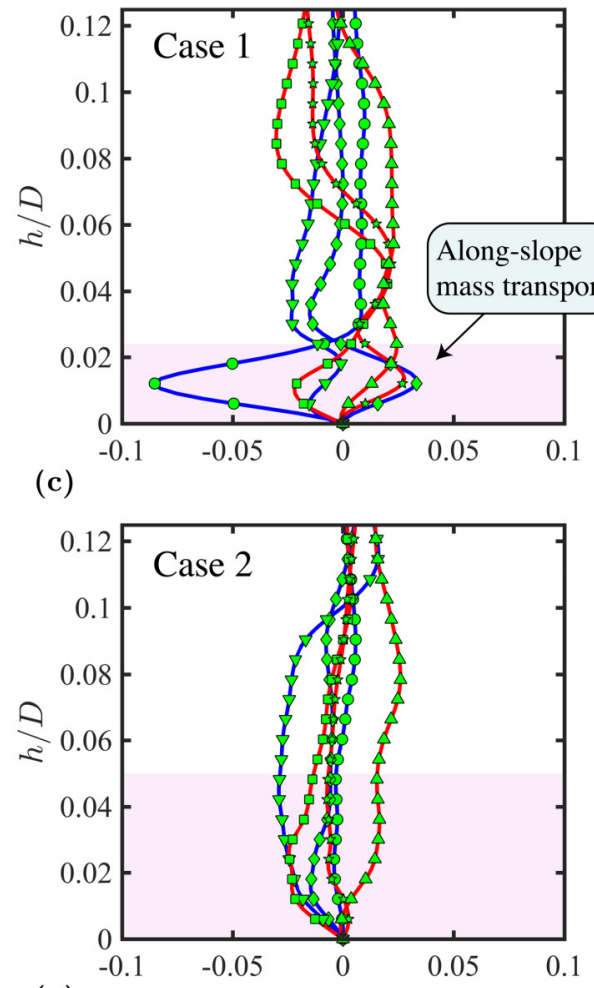

(e)

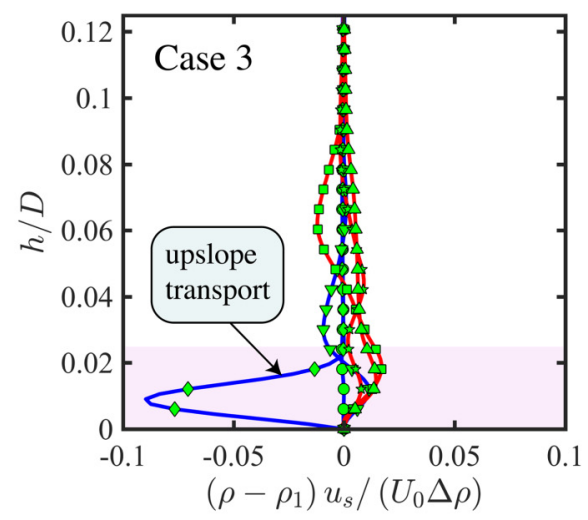

(b) $\quad(x, z)_{\star \star} / D=(-37.5,0.2)$

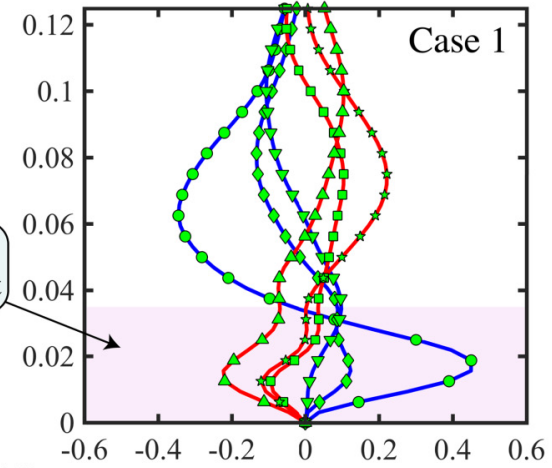

(d)

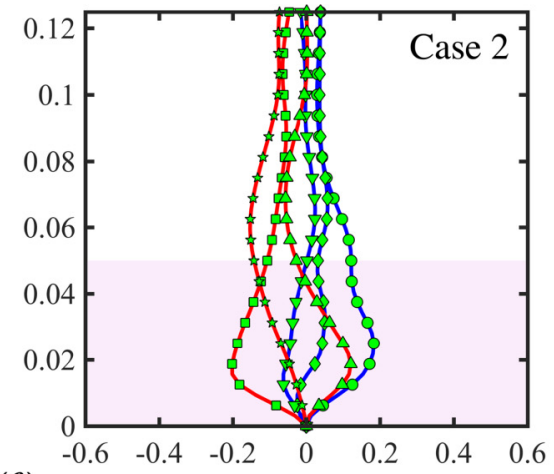

(f)

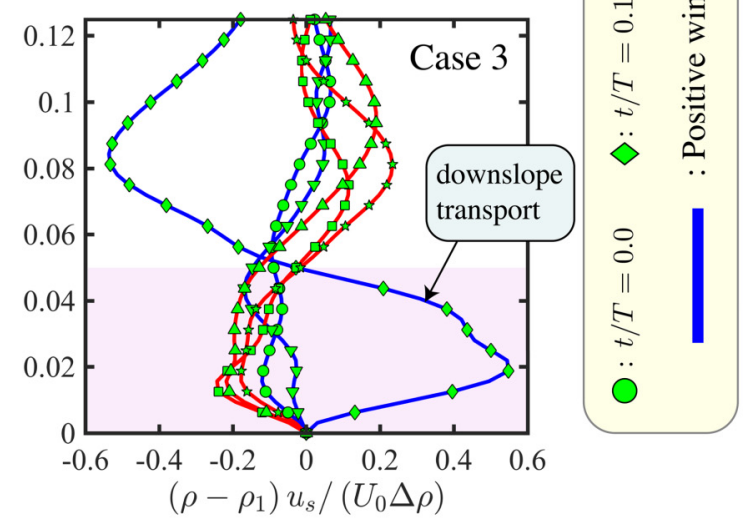

FIG. 7. BBL mass transport along the sloping bottom over the diurnal phase for the three different cases. The along-slope flow is determined as $u_{s} \equiv\langle\rangle_{\mathrm{sw}} \cdot \hat{s}$, where $\hat{s}$ is the along-slope normalized vector (positive downslope). $h / D$ corresponds to the nondimensional height relative to the local bottom boundary. The alongslope mass transport, (a),(c),(e) above and (b),(d),(f) below the pycnocline, respectively. Fields are shown in nondimensional form.

\section{Along-slope mass transport}

We now examine the BBLT as a function of the diurnal phase and space. Figure 7 summarizes the diurnal evolution of the along-slope BBLT, $\left(\rho-\rho_{1}\right) u_{s} /\left(U_{0} \Delta \rho\right)$, for each case at two different positions. The shallower spot [Figs. 7(a), 7(c), 7(e)] is located above the equilibrium pycnocline 
depth, $(x, z)_{*} / D=(-52.5,0.61)$, while the deeper spot [Figs. 7(b), 7(d), 7(f)] is located below the pycnocline, $(x, z)_{* *} / D=(-37.5,0.2)$. Each profile in Fig. 7 corresponds to a different diurnal phase. The line colors define the wind direction (positive winds in blue, negative winds in red), while the shaded area denotes the bottom layer.

The BBLT in cases 1 (QL-V2H1) and 3 (NL-V2H1) shows similar features. In both cases, the baroclinic flows concentrate the mass transport near the bottom [shaded areas in Figs. 7(a), 7(b), 7(e), and 7(f)]. In case 1, the midlayer flow reaching the sloping boundary [Figs. 2(a), 7(a), and 7(b)] is split into two branches. The upper branch drives the upslope mass transport [Fig. 7(a)], whereas the lower branch drives a more vigorous downslope mass transport. The along-slope transport is maximized when the flow is wind free $(t / T=0$ on the left sloping bottom and $t / T=0.5$ on the right sloping bottom) and it decays when the wind intensity reaches its maximum. Nevertheless, in case 3, the downslope transport is concentrated in a substantially thicker layer than the upslope transport [Figs. 7(e) and 7(f)]. The intensification of the downslope transport may be related to the fact that the midlayer flow reaches the sloping boundary while the wind is reaching its maximum magnitude at $t / T=0.125$ on the left slope and $t / T=0.625$ on the right slope. For these wind phases, the downslope transport is wind reinforced.

By contrast, case 2 (QL-V1H1) shows an along-slope mass transport that is significantly weaker than that for cases 1 and 3. Furthermore, it does not show baroclinic dynamics near the bottom. We attribute a drop in the BBLT to the absence of a midlayer flow toward and from the sloping boundaries in the QL-V1H1 mode. The latter is a key mechanism that reinforces the along-slope transport in both directions.

\section{Bottom shear stress}

BBL flows are a relevant mechanism for sediment resuspension [24,37,38,55-57] and enrichment of waters with benthic biogeochemical properties [23,25]. Figure 8 shows and contrasts the diurnal variation of the wind-induced surface stress, $|\tau| / \tau_{0}$ [Fig. 8(a)], the average bottom shear stress, $\left\langle\left|\tau_{b}\right|\right\rangle \equiv \mu\left\langle\left|\frac{\partial u}{\partial n}\right|\right\rangle$ [Fig. 8(b)], and the average bed shear stress fluctuations, $\tau_{\mathrm{rms}} \equiv \mu\left\langle\left|\frac{\partial \sqrt{T K E}}{\partial n}\right|\right\rangle$ [Fig. 8(c)], over the basin's bottom for each case; $\mu$ is the dynamic viscosity. In case 1 (QL-V2H1), the maximum $\left\langle\left|\tau_{b}\right|\right\rangle$ is predicted at the times when the wind stress is null. In case 3 (NL-V2H1), the maximum of $\left\langle\left|\tau_{b}\right|\right\rangle$ is delayed by $1 / 8$ of a period with respect to that for case 1 . Case 2 (QL-V1H1) is characterized by the lowest values of $\left|\tau_{b}\right|$, and its highest magnitudes are delayed by $1 / 8$ of a period with respect to the maximum values of $|\tau|$. Results show that for cases 1 and 3, the maximum values of $\tau_{\text {rms }}$ are well correlated with those of $\tau_{b}$.

In case 3 , the peaks of $\left\langle\left|\tau_{b}\right|\right\rangle$ and $\tau_{\mathrm{rms}}$ at $t / T \approx 0.1$ and 0.6 are associated with the times when the undular bore interacts with the inclined bottom (see the impact of the internal wave front hitting the left sloping bottom in Fig. 6). In case 1, however, $\left\langle\left|\tau_{b}\right|\right\rangle$ and $\tau_{\text {rms }}$ reach their highest magnitudes around $t / T \approx 0.0$ and 0.5 , when the maximum magnitude of the horizontal currents (horizontal isopycnals) is achieved [Fig. 2(e)]. At these wind phases, the horizontal flow starts interacting with the sloping bottom and its magnitude reduces. The latter leads to a decrease in $\tau_{b}$ and $\tau_{\text {rms }}$ until these quantities reach their minimum magnitudes. This occurs when the maximum midlayer stretching and squeezing occurs on the sloping regions at $t / T \approx 0.25$ and 0.75 . This is not quite the scenario for case 2 , as $\tau_{\mathrm{rms}}$ is delayed by $1 / 8$ from the maximum magnitudes of $\tau_{b}$. The maximum $\tau_{\mathrm{rms}}$ is obtained when the fluid is wind free at $t / T=0$ and 0.5 , while the maximum $\tau_{b}$ is reached during the flow acceleration phase along slope, $0.25 \leqslant t / T<0.5$ and $0.75 \leqslant t / T<1$. The results show that case 2 has the weakest $\tau_{b}$ and $\tau_{\text {rms }}$, while the highest magnitudes are found in case 1 .

\section{Residual flow}

An inviscid, immiscible, linear basin-scale internal wave with period $T$ that is in phase with the wind forcing leads to a null net transport at the end of its cycle. By contrast, viscous, miscible, advective flows admit residual flows at the end of the wind-forcing cycle, $T$. The diurnally averaged 

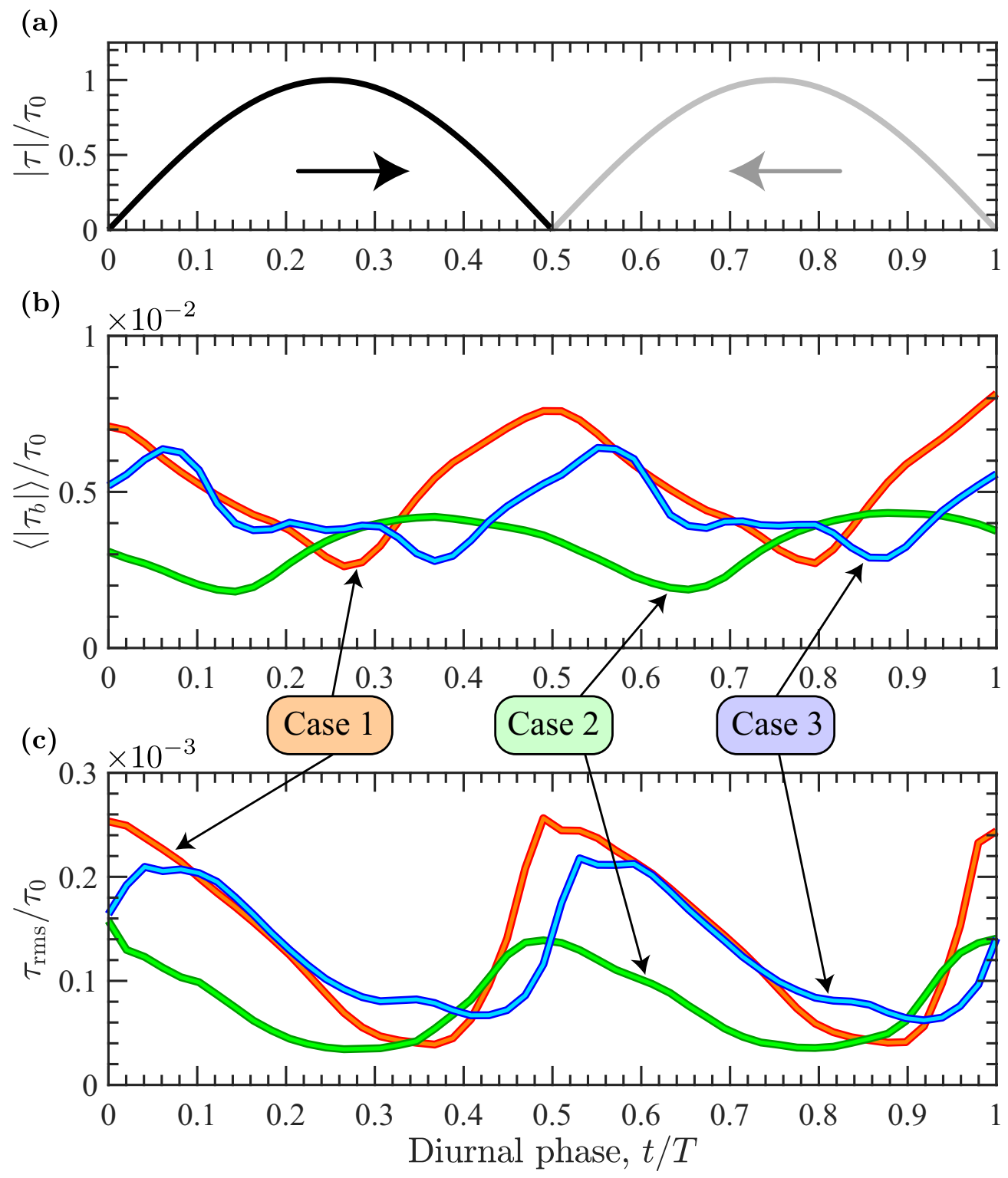

FIG. 8. (a) Magnitude and direction of the surface stress, (b) average bottom shear stress, and (c) average bed shear stress fluctuations over the diurnal phase for each experiment.

velocity field thus provides a residual (secondary) circulation [e.g., 27,29] resulting from coupling the wind-driven flow and the basin-scale internal waves. Although the residual flow might vary seasonally due to changes in the background stratification and forcing, here we assume that it is invariant in time as long as the resonance regime lasts.

Figure 9 shows the diurnally averaged $(0 \leqslant t / T \leqslant 1)$ density $\langle\rho\rangle_{\mathrm{sw}, T}$ (left panels) and streamwise velocity $\langle u\rangle_{\mathrm{sw}, T}$ along with the streamlines (right panels) for each case. We denote these quantities as the residual fields. The density $\langle\rho\rangle_{\mathrm{sw}, T}$ has a symmetric distribution with respect to the vertical axis that bisects the domain at $x=0,\langle\rho\rangle_{\mathrm{sw}, T}(x<0, z)=\langle\rho\rangle_{\mathrm{sw}, T}(x>0, z)$. On the other hand, $\langle u\rangle_{\mathrm{sw}, T}$ has an antisymmetric distribution with respect to the vertical plane at 

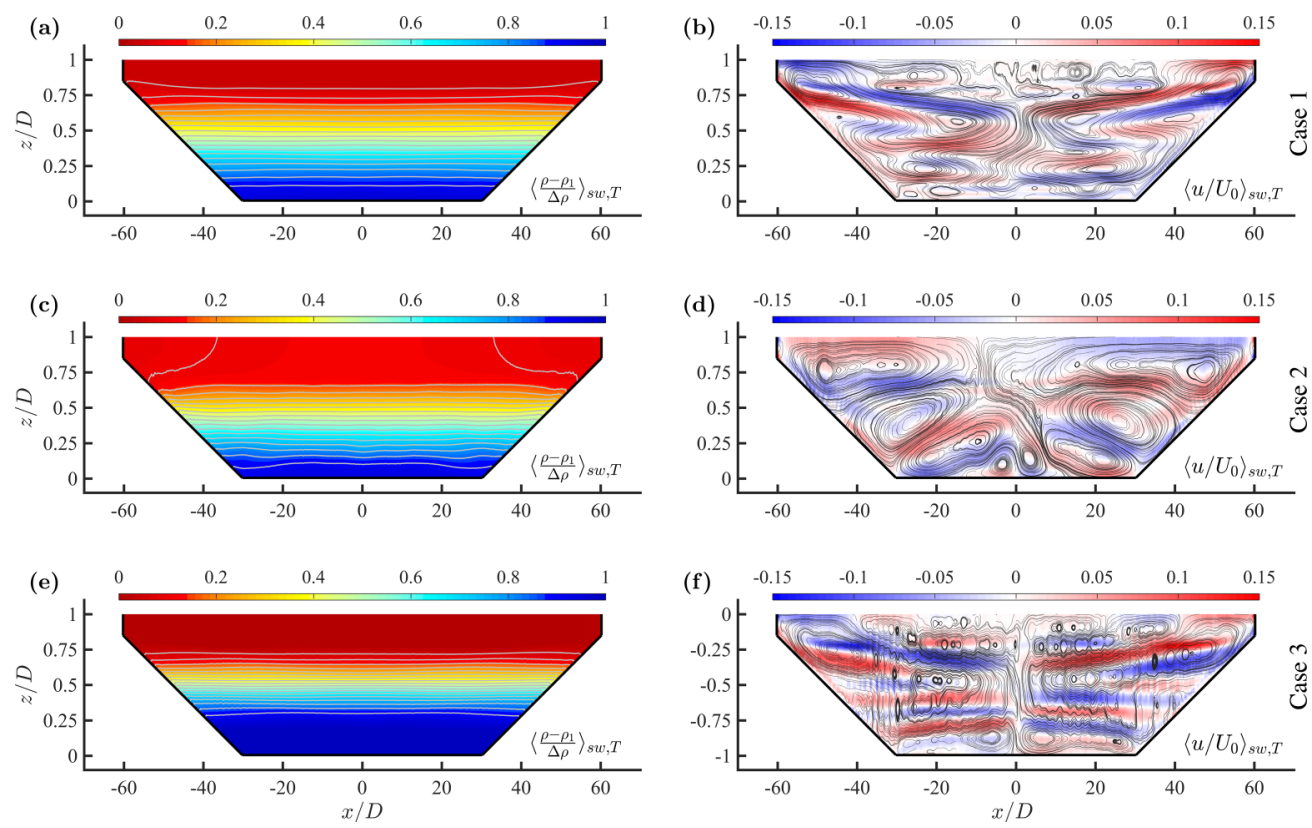

FIG. 9. (a),(c),(e) Spanwise and time-averaged density along with isopycnals for each case. (b),(d),(f) Residual streamwise flow along with streamlines for each case.

$x=0,\langle u\rangle_{\mathrm{sw}, T}(x<0, z)=-\langle u\rangle_{\mathrm{sw}, T}(x>0, z)$. In cases $1(\mathrm{QL}-\mathrm{V} 2 \mathrm{H} 1)$ and $3(\mathrm{NL}-\mathrm{V} 2 \mathrm{H} 1),\langle u\rangle_{\mathrm{sw}, T}$ and $\langle\rho\rangle_{\text {sw,T }}$ show similar spatial distributions [Figs. 9(a), 9(b), 9(e), and 9(f)]. In both cases, the isopycnals are slightly stretched near the sloping boundaries, while being slightly squeezed at the center of the basin.

Case 2 (QL-V1H1), by contrast, shows that the isopycnals are squeezed near the sloping boundaries and slightly lifted in the center of the basin [Fig. 9(c)], with well-defined clockwise and counterclockwise circulation cells in the upper layer [Fig. 9(d)]. The residual flow along the slopes has the same features as that obtained by Nakayama et al. [27,29] via laboratory experiments of internal wave shoaling over uniform slopes in a two-layer stratified basin. Yet, unlike Nakayama et al. [27,29], our results reveal that the residual flow is characterized by an array of circulation cells. The largest cells encompass almost half of the domain in the upper waters, whereas deeper waters are associated with smaller cells.

\section{Horizontal mass exchange}

We quantify the rate of mass exchange between the shallows and the interior basin due to horizontal flows as a function of time and space [Fig. 10(a)]. We first integrate in time the mass flux per unit width, $F_{\text {ex }}$, exchanged between two volumes vertically bisected at a distance $x_{s}$ from the left lateral boundary. This integration provides the exchanged mass, $M_{\mathrm{ex}}$, over a time window $\Delta t=t-t_{0} \leqslant T$ at a position $x_{s}$. By dividing $M_{\mathrm{ex}}$ by the mass contained in the nearshore volume, $M$ [Fig. 10(a)], we obtain the nondimensional rate of exchange of nearshore waters, $R_{\text {ex }}$. The latter ratio is a function of the diurnal phase and the distance from the lateral boundary, $x_{s}$.

Figures 10(b)-10(d) map $R_{\mathrm{ex}}\left(t, x_{s}\right)$ for each experiment. The horizontal axis shows $R_{\mathrm{ex}}$ at a given position $x_{s} / D$, while the vertical axis shows its evolution over the diurnal phase, $t / D$. Bold lines highlight regions that are partially $(25 \%, 50 \%$, and $75 \%)$ and fully $(100 \%)$ flushed. The results show that very shallow waters $\left(x_{s} / D<5\right)$ can be fully flushed and exchanged with interior waters 

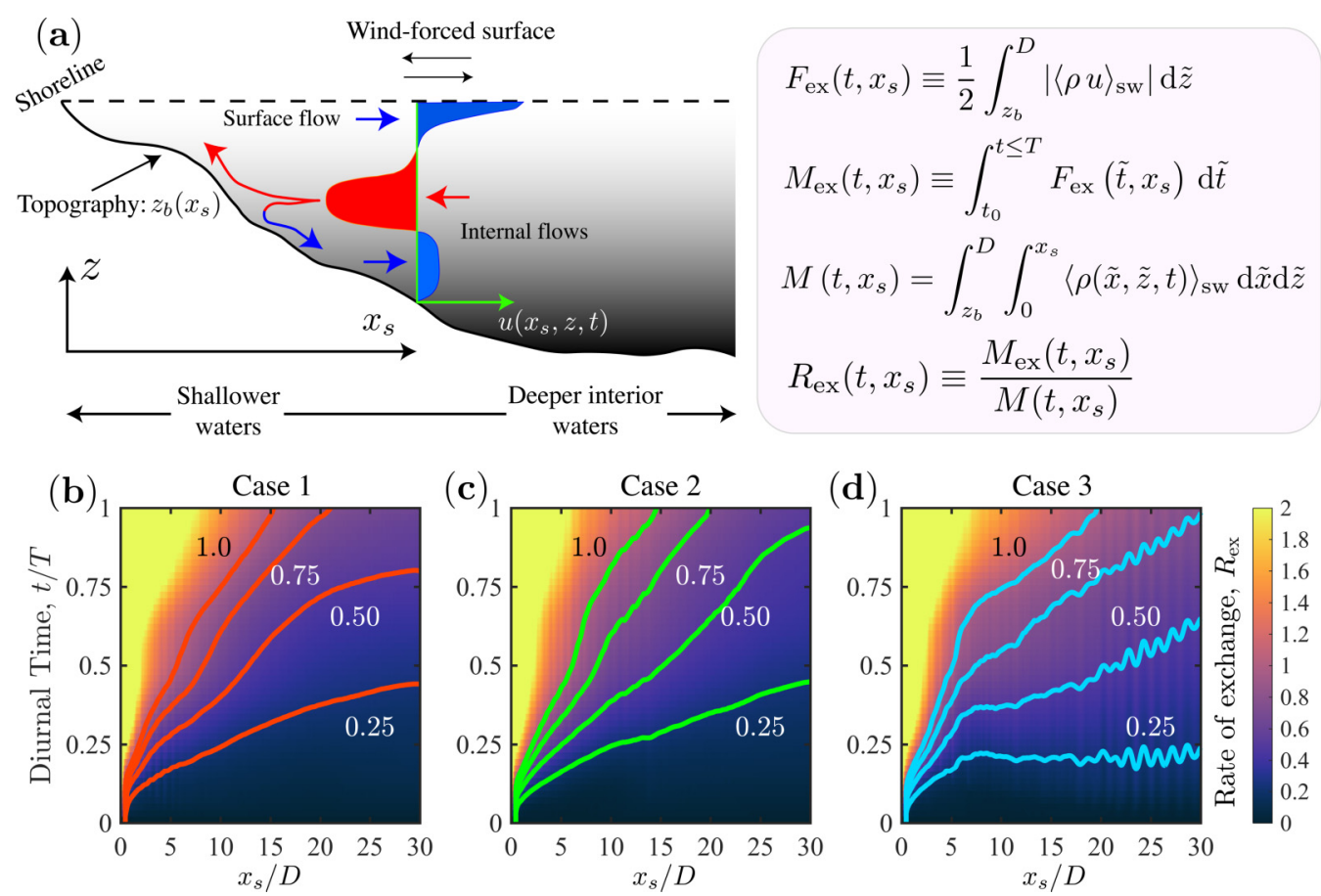

FIG. 10. (a) Schematics of wind-driven exchange flows and integral quantities. (b)-(d) Rate of cross-shore mass exchange, $R_{\mathrm{ex}}$, between nearshore and interior regions during the diurnal phase for each case, respectively. Color lines illustrate four specific exchange rates $(25 \%, 50 \%, 75 \%$, and $100 \%)$ from the left boundary to a distance $x_{s} / D$ offshore as a function of $t / T$.

over T/2 (e.g., half of a day). We found that cases 1 (QL-V2H1) and 2 (QL-V1H1) can fully exchange and thus renew waters up to a distance of $\sim 15 D$ from the left shore over a diurnal phase [see the red and green curves in Figs. 10(b) and 10(c) labeled "1" in black]; this means $63 \%$ of the sloping volume is flushed. Case 3 (NL-V2H1), however, can exchange and flush waters up to a distance $\sim 20 D$ over $T$ [see the cyan curve in Fig. 10(d) labeled "1" in black]; this means $75 \%$ of the sloping volume is flushed. The bold curves in Figs. 10(b)-10(d) show that $R_{\mathrm{ex}}$ is, in general, similar in space and time for the $\mathrm{V} 1 \mathrm{H} 1$ and $\mathrm{V} 2 \mathrm{H} 1$ modes. By contrast, $R_{\mathrm{ex}}$ in case 3 suffers a substantial change around $x_{s}=5 D$; for a given time and position, case 3 shows higher values of $R_{\mathrm{ex}}$ than do cases 1 and 2. For instance, compare curves $R_{\mathrm{ex}}=0.25$ in Figs. 10(b)-10(d).

We finally assess the mass exchange between the shallow and interior regions due to the diurnal residual flow. From the residual fields $\langle u\rangle_{\mathrm{sw}, T}$ and $\langle\rho\rangle_{\mathrm{sw}, T}$ (Fig. 9), we compute for each case the flushing timescale in terms of the diurnal wind period, $T_{F} / T$, as a function of $x_{S} / D$ (Fig. 11). The results show that the residual flow for case 2 is less efficient in flushing nearshore waters, especially close to the lateral boundaries where the secondary circulation is extremely weak compared with cases 1 and 3 (Fig. 11; green curve near $x_{s} / D \approx 2$ ).

The green curve of case 2 (Fig. 11) is generally above the other two curves for $0 \leqslant x_{s} / D \leqslant$ 30 , indicating that its flushing time is the longest over the sloping boundary. However, this trend changes abruptly near the transition to the flat interior basin for $30 \leqslant x_{s} / D \leqslant 36$. In this region, case 2 has the lowest $T_{F} / T$ and the strongest residual circulation. At $x_{S} / D \approx 40, T_{F} / T$ increases asymptotically due to a strong drop in horizontal transport near the basin's center. In this interior region, for $40 \leqslant x_{s} / D \leqslant 50$, case 3 and case 2 have the shortest and the longest flushing times, respectively. 


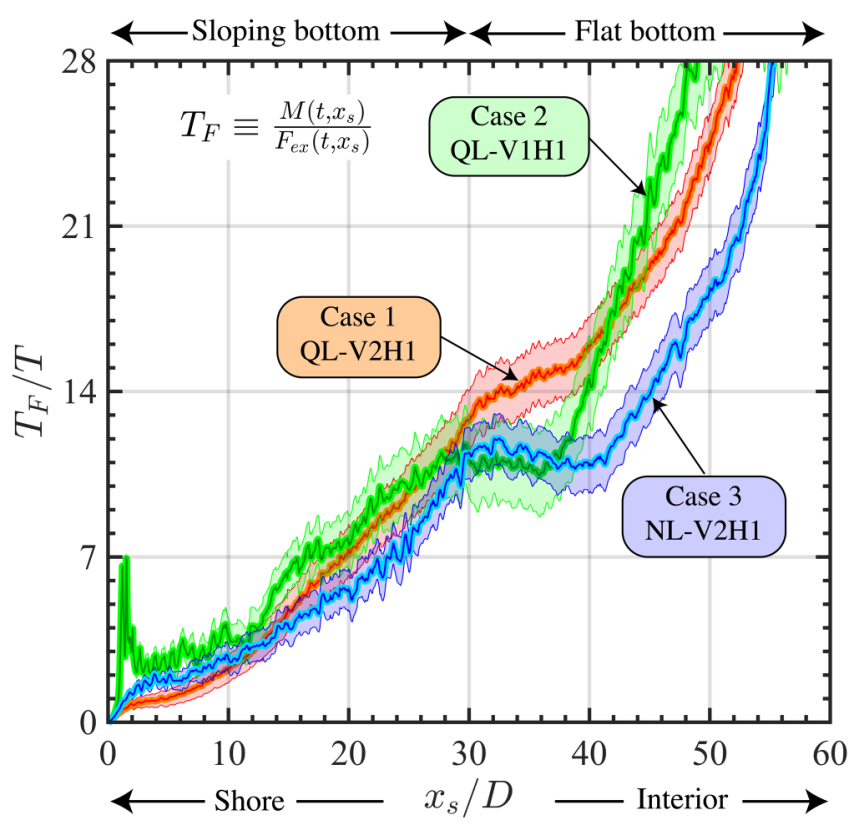

FIG. 11. Flushing timescale $T_{F} / T$ (in units of day) based on the residual cross-shore mass transport (Fig. 9) for each case along its standard deviation as a function of the distance from the lateral boundary $x_{s} / D .60 D$ is half of the horizontal (main axis) length scale of the basin.

\section{DISCUSSION AND SUMMARY}

Although the resonance of basin-scale internal waves is not frequently observed, it is not an unusual hydrodynamic regime in stratified lakes. As suggested by Mortimer [1], of all the internal waves that are theoretically possible in stratified lakes, the one most commonly amplified and set in motion is the basin-scale internal mode, also known as the uninodal seiche [9]. The above implicitly suggests that this normal mode could be regarded as the canonical resonant response of stratified basins to wind [1]. After Mortimer's work, several studies have reported wind-induced resonant regimes in lakes [1,7,10-16]. However, these observations show that the first vertical seiche (V1H1) is not the only one susceptible to wind-driven amplification. In smaller basins, uninodal horizontal modes with higher vertical modes (e.g., the V2H1 and V3H1 modes) can also be found to resonate with diurnal winds $[13,14]$.

As shown by Antenucci and Imberger [12], and most recently by Woolway and Simpson [15], lakes subject to diurnal winds may reach resonance regimes at different periods of the annual thermal cycle. With annual information of the stratification and the basin's topography, one might use Sturm-Liouville theory to identify periods when diurnal winds may amplify basin-scale normal modes whose frequencies are near $24 \mathrm{~h}$ [41,42]. Wind-induced resonance of basin-scale internal motions might occur more frequently than we reported. For instance, temperate lakes embedded in valleys and mountain regions subject to local diurnal (day and night) breezes [58] are potential candidates for basin-scale internal waves under resonance conditions.

In this study, we achieved resonance regimes by running simulations over several cycles before obtaining periodic flows with frequencies near the wind frequency. Longer numerical experiments, however, might result in basin-scale internal waves that deviate from resonance conditions due to vertical mixing. The latter affects the background density distribution and thus the dispersion relationship of the internal waves. Laboratory experiments show that significant modal amplification occurs when the ratio of the basin-scale internal wave frequency, $\omega_{r}$, to the forcing frequency, $\omega_{F}$, is $\omega_{r} / \omega_{F} \in[0.9,1.1]$. On the other hand, when $\omega_{r} / \omega_{F}<0.8$, a substantial decrease in the wave 
amplitude is observed [5-8]. To characterize how periodically wind-forced stratified basins reach or escape from resonance, we require a more in-depth examination of the system's evolution.

As shown in the present study, even mild periodic winds can magnify the amplitude of basin-scale internal waves and thus trigger nonlinear wave regimes $[6,7,43,59]$ that intensify on sloping regions $[30,44,57]$. Under a resonance regime, the periodic interaction between vigorous basin-scale internal waves and sloping boundaries supports an active bottom boundary layer transport, BBLT [22], that in turn can enhance sediment and nutrient resuspension $[25,26,57,60]$ and the exchange of dissolved gases between deep and shallow regions [23]. The features of the BBLT described here have been commonly observed in the context of nonlinear internal waves interacting with cross-shelf sloping boundaries [e.g., 57,61], yet observations of the BBLT in lakes under resonance are rather rare [18].

Carr et al. [31] investigated the shoaling of second-mode solitarylike waves via laboratory and numerical experiments generated using a lock-release method in a still, three-layer stratified fluid. By contrast, the second-mode nonlinear internal waves in case 3 (NL-V2H1) result from a periodic forcing, and they coexist with a wind-driven basin-scale circulation. Although the mechanisms leading to the second-mode solitarylike waves differ, in both studies, the nonlinear internal waves have similar degeneration processes as they approach the sloping boundary. However, for the same bottom slope, $s \approx 3 \%$, the second-mode nonlinear internal waves (in case 3) present a robust wave reflection, while the similar internal solitarylike waves in Carr et al. [31] show no wave reflection. This last result suggests that the wind-driven baroclinic circulation might be crucial for supporting the periodic propagation of the nonlinear internal waves from side to side in the stratified basin.

In the present study, the slope steepness is not varied. We adopt a mild slope, $s \approx 3 \%$, that is characteristic of many shallow lakes. In this scenario, internal waves degenerate along the slope, which enhances turbulence and vertical mixing. However, previous studies show that, as the slopes steepen, the degeneration of internal waves becomes weaker and waves can reflect more energy [31,52]. This implies less mixing and less dissipation of kinetic energy on the sloping boundaries $[30,44]$. We speculate that stratified waterbodies with steeper slopes would suffer weaker changes in the background stratification, allowing resonance regimes to last longer before mixing allows an escape from resonance. Additionally, steeper slopes might reduce along-slope transport and the bottom shear stress due to the restoring effect of stratification. However, since steeper slopes also reduce the volume of the shallow regions, the flushing time of nearshore waters might be reduced.

Our first numerical experiment (case 1) provides a global picture of the hydrodynamics of Lake Alpnach, Switzerland, a stratified lake that experiences resonance regimes during the stratified season. The residual flow obtained for case 1 [Fig. 9(b)] can entirely flush the sloping bottom region in less than 14 wind cycles. This result agrees with the in situ experiments of Goudsmit et al. [22].

\section{CONCLUSIONS}

In the present study, we found that the along-slope bottom boundary layer transport (BBLT) is sensitive to the resonant basin-scale internal mode and the wave dynamics. The results show that the BBLT associated with the nonlinear, second-mode, undular bore in case 3 (NL-V2H1) [e.g., $10,47,62]$ is more vigorous than the BBLT observed in the quasilinear, second-mode, basin-scale internal seiche in case 1 (QL-V2H1). The first vertical basin-scale internal seiche in case 2 (QL$\mathrm{V} 1 \mathrm{H} 1)$ is associated with the weakest BBLT.

Differences in the circulation pattern across stratified basins have strong effects on the rate of mass exchange and flushing timescales of nearshore waters. Over the diurnal wind cycle, we found that the horizontal mass transport in the region with the sloping bottom is comparable for the two QL-V1H1 and QL-V2H1 modes. Both modal responses can fully flush $63 \%$ of the water contained in the wedgelike volume of the stratified basins. However, the mass transport attributed to NL-V2H1 is stronger and can flush $75 \%$ of the water contained in the same volume over the same time.

The net horizontal transport at the end of the wind-forcing cycle is not zero. This result is a consequence of the phase mismatch between the wind-driven nonlinear transport in an enclosed basin and the amplified basin-scale internal waves. One of the implications of the last regime is 
the existence of a residual flow that affects the mass distribution in the stratified basin at the end of the forcing cycle. We found that the residual circulation in case 1 can entirely flush the sloping bottom region in less than 14 wind cycles. On the other hand, due to the lack of residual circulation in the very shallow regions, a basin that hosts the QL-V1H1 mode is characterized by long flushing timescales for shallow waters. We also found that the basin hosting the NL-V2H1 mode has the most vigorous residual flow and, in general, the shortest flushing timescales.

\section{ACKNOWLEDGMENTS}

This work was carried out during a visit by G.C. to EPFL under the ENAC Visiting Professor Program (Grant No. CF 0233). We are thankful to P. A. Davies and the authors of the paper "Shoaling mode-2 internal solitary-like waves" [31] who provided valuable information regarding experimental and numerical results of second-mode solitarylike waves interacting with sloping boundaries. Finally, we are thankful for the constructive feedback from the anonymous reviewers.

[1] C. H. Mortimer, The resonant response of stratified lakes to wind, Schweizer. Zeitschrift Hydrol. 15, 94 (1953).

[2] R. H. Spigel and J. Imberger, The classification of mixed-layer dynamics of lakes of small to medium size, J. Phys. Oceanogr. 10, 1104 (1980).

[3] J. Imberger, in Physical Processes in Lakes and Oceans, edited by J. Imberger (American Geophysical Union,1998), pp. 1-17.

[4] A. Wüest and A. Lorke, Small-scale hydrodynamics in lakes, Annu. Rev. Fluid Mech. 35, 373 (2003).

[5] G. W. Wake, E. J. Hopfinger, and G. N. Ivey, Experimental study on resonantly forced interfacial waves in a stratified circular cylindrical basin, J. Fluid Mech. 582, 203 (2007).

[6] L. Boegman and G. N. Ivey, The dynamics of internal wave resonance in periodically forced narrow basins, J. Geophys. Res. 117, C11002 (2012).

[7] C. Rozas, A. de la Fuente, H. Ulloa, P. Davies, and Y. Niño, Quantifying the effect of wind on internal wave resonance in lake Villarrica, Chile, Environ. Fluid Mech. 14, 849 (2014).

[8] P. Rojas, H. N. Ulloa, and Y. Niño, Experimental study on periodically forced interfacial gravity waves in a rotating cylindrical basin (unpublished).

[9] F. A. Forel, Première étude sur les seiches, Bull. Soc. Vaudoise Sci. Nat. 12, 213 (1873).

[10] S. A. Thorpe, Near-resonant forcing in a shallow two-layer fluid: A model for the internal surge in Loch Ness? J. Fluid Mech. 63, 509 (1974).

[11] M. Münnich, A. Wüest, and D. M. Imboden, Observations of the second vertical mode of the internal seiche in an alpine lake, Limnol. Oceanogr. 37, 1705 (1992).

[12] J. P. Antenucci and J. Imberger, The seasonal evolution of wind/internal wave resonance in Lake Kinneret, Limnol. Oceanogr. 48, 2055 (2003).

[13] J. Vidal, X. Casamitjana, J. Colomer, and T. Serra, The internal wave field in Sau Reservoir: Observation and modeling of a third vertical mode, Limnol. Oceanogr. 50, 1326 (2005).

[14] A. Pannard, B. E. Beisner, D. F. Bird, J. Braun, D. Planas, and M. Bormans, Recurrent internal waves in a small lake: Potential ecological consequences for metalimnetic phytoplankton populations, Limnol. Oceanogr. Fluids Environ. 1, 91 (2011).

[15] R. I. Woolway and J. H. Simpson, Energy input and dissipation in a temperate lake during the spring transition, Ocean Dyn. 67, 959 (2017).

[16] A. Posada-Bedoya, A. Gómez-Giraldo, and R. R. Botero, Preliminary characterization of the dominant baroclinic modes of a tropical Andean reservoir during a dry period, Rev. Acad. Colomb. Cienc. Ex. Fis. Nat. 43, 297 (2019).

[17] B. C. Kenney, Lake surface fluctuations and the mass flow through the narrows of Lake Winnipeg, J. Geophys. Res. 84, 1225 (1979). 
[18] C. Lorrai, L. Umlauf, J. K. Becherer, A. Lorke, and A. Wüest, Boundary mixing in lakes: 2. Combined effects of shear- and convectively induced turbulence on basin-scale mixing, J. Geophys. Res. 116, C10018 (2011).

[19] S. M. Henderson and B. R. Deemer, Vertical propagation of lakewide internal waves, Geophys. Res. Lett. 39, L06405 (2012).

[20] H. N. Ulloa, G. Constantinescu, K. Chang, D. Horna-Munoz, O. S. Steiner, D. Bouffard, and A. Wüest, Hydrodynamics of a periodically wind-forced small and narrow stratified basin: A large-eddy simulation experiment, Environ. Fluid Mech. 19, 667 (2019).

[21] D. J. Wain, M. S. Kohn, J. A. Scanlon, and C. R. Rehmann, Internal wave-driven transport of fluid away from the boundary of a lake, Limnol. Oceanogr. 58, 429 (2013).

[22] G.-H. Goudsmit, F. Peeters, M. Gloor, and A. Wüest, Boundary versus internal diapycnal mixing in stratified natural waters, J. Geophys. Res. Ocean. 102, 27903 (1997).

[23] B. Müller, L. D. Bryant, A. Matzinger, and A. Wüest, Hypolimnetic oxygen depletion in eutrophic lakes, Environ. Sci. Technol. 46, 9964 (2012).

[24] M. Gloor, A. Wüest, and M. Münnich, Benthic boundary mixing and resuspension induced by internal seiches, Hydrobiologia 284, 59 (1994).

[25] B. R. Deemer, S. M. Henderson, and J. A. Harrison, Chemical mixing in the bottom boundary layer of a eutrophic reservoir: The effects of internal seiching on nitrogen dynamics, Limnol. Oceanogr. 60, 1642 (2015).

[26] M. Gloor, A. Wüest, and D. M. Imboden, Dynamics of mixed bottom boundary layers and its implications for diapycnal transport in a stratified, natural water basin, J. Geophys. Res. Ocean. 105, 8629 (2000).

[27] K. Nakayama and J. Imberger, Residual circulation due to internal waves shoaling on a slope, Limnol. Oceanogr. 55, 1009 (2010).

[28] T. Sakai and L. G. Redekopp, A parametric study of the generation and degeneration of wind-forced long internal waves in narrow lakes, J. Fluid Mech. 645, 315 (2010).

[29] K. Nakayama, T. Shintani, K. Kokubo, T. Kakinuma, Y. Maruya, K. Komai, and T. Okada, Residual currents over a uniform slope due to breaking of internal waves in a two-layer system, J. Geophys. Res. 117, C10002 (2012).

[30] R. S. Arthur and O. B. Fringer, Transport by breaking internal gravity waves on slopes, J. Fluid Mech. 789, 93 (2016).

[31] M. Carr, M. Stastna, P. A. Davies, and K. J. V. de Wal, Shoaling mode-2 internal solitary-like waves, J. Fluid Mech. 879, 604 (2019).

[32] C. D. Pierce and P. Moin, Progress-variable approach for large-eddy simulation of turbulent combustion, Ph.D. thesis, Standford University, 2001.

[33] S. K. Ooi, G. Constantinescu, and L. Weber, Numerical simulations of lock-exchange compositional gravity current, J. Fluid Mech. 635, 361 (2009).

[34] Z. Borden, E. Meiburg, and G. Constantinescu, Internal bores: An improved model via a detailed analysis of the energy budget, J. Fluid Mech. 703, 279 (2012).

[35] K. Mahesh, G. Constantinescu, and P. Moin, A numerical method for large-eddy simulation in complex geometries, J. Comput. Phys. 197, 215 (2004).

[36] S. K. Ooi, G. Constantinescu, and L. Weber, A numerical study of intrusive compositional gravity currents, Phys. Fluids 19, 076602 (2007).

[37] K. Steenhauer, T. Tokyay, and G. Constantinescu, Dynamics and structure of planar gravity currents propagating down an inclined surface, Phys. Fluids 29, 036604 (2017).

[38] T. Tokyay and G. Constantinescu, The effects of a submerged non-erodible triangular obstacle on bottom propagating gravity currents, Phys. Fluids 27, 056601 (2015).

[39] G. la Forgia, T. Tokyay, C. Adduce, and G. Constantinescu, Numerical investigation of breaking internal solitary waves, Phys. Rev. Fluids 3, 104801 (2018).

[40] T. Sommer, F. Danza, J. Berg, A. Sengupta, G. Constantinescu, T. Tokyay, H. Bürgmann, Y. Dressler, O. Sepúlveda Steiner, C. J. Schubert, M. Tonolla, and A. Wüest, Bacteria-induced mixing in natural waters, Geophys. Res. Lett. 44, 9424 (2017). 
[41] P. D. Fricker and H. M. Nepf, Bathymetry, stratification, and internal seiche structure, J. Geophys. Res. 105, 14237 (2000).

[42] Y. E. Imam, Modal decomposition of the baroclinic response to wind in elongated lakes, Ph.D. thesis, University of British Columbia, 2011.

[43] D. A. Horn, J. Imberger, and G. N. Ivey, The degeneration of large-scale interfacial gravity waves in lakes, J. Fluid Mech. 434, 181 (2001).

[44] L. Boegman, G. Ivey, and J. Imberger, The degeneration of internal waves in lakes with sloping topography, Limnol. Oceanogr. 50, 1620 (2005).

[45] K. R. Helfrich and W. K. Melville, Long nonlinear internal waves, Annu. Rev. Fluid Mech. 38, 395 (2006).

[46] H. N. Ulloa, A. de la Fuente, and Y. Niño, An experimental study of the free evolution of rotating, nonlinear internal gravity waves in a two-layer stratified fluid, J. Fluid Mech. 742, 308 (2014).

[47] G. A. El, R. H. J. Grimshaw, and W. K. Tiong, Transformation of a shoaling undular bore, J. Fluid Mech. 709, 371 (2012).

[48] S. A. Thorpe, Asymmetry of the internal seiche in Loch Ness, Nature (London) 231, 306 (1971).

[49] J. Olsthoorn, A. Baglaenko, and M. Stastna, Analysis of asymmetries in propagating mode-2 waves, Nonlinear Proc. Geophys. 20, 59 (2013).

[50] M. Carr, P. A. Davies, and R. P. Hoebers, Experiments on the structure and stability of mode-2 internal solitary-like waves propagating on an offset pycnocline, Phys. Fluids 27, 046602 (2015).

[51] D. Deepwell and M. Stastna, Mass transport by mode-2 internal solitary-like waves, Phys. Fluids 28, 056606 (2016).

[52] P. Aghsaee, L. Boegman, and K. G. Lamb, Breaking of shoaling internal solitary waves, J. Fluid Mech. 659, 289 (2010).

[53] B. R. Sutherland, K. J. Barrett, and G. N. Ivey, Shoaling internal solitary waves, J. Geophys. Res.: Oceans 118, 4111 (2013).

[54] R. S. Arthur and O. B. Fringer, The dynamics of breaking internal solitary waves on slopes, J. Fluid Mech. 761, 360 (2014).

[55] T. Tokyay, G. Constantinescu, and E. Meiburg, Tail structure and bed friction velocity distribution of gravity currents propagating over an array of obstacles, J. Fluid Mech. 694, 252 (2012).

[56] T. Tokyay, G. Constantinescu, and E. Meiburg, Lock-exchange gravity currents with a low volume of release propagating over an array of obstacles, J. Geophys. Res.: Oceans 119, 2752 (2014).

[57] L. Boegman and M. Stastna, Sediment resuspension and transport by internal solitary waves, Annu. Rev. Fluid Mech. 51, 129 (2019).

[58] D. Zardi and C. D. Whiteman, Springer Atmospheric Sciences (Springer, Netherlands, 2012).

[59] H. N. Ulloa, K. B. Winters, A. de la Fuente, and Y. Niño, Degeneration of internal Kelvin waves in a continuous two-layer stratification, J. Fluid Mech. 777, 68 (2015).

[60] P. McKinney, J. Austin, and G. Fai, The wind-driven formation of cross-shelf sediment plumes in a large lake, Limnol. Oceanogr. 64, 1309 (2019).

[61] J. M. Klymak and J. N. Moum, Internal solitary waves of elevation advancing on a shoaling shelf, Geophys. Res. Lett. 30, 2045 (2003).

[62] A. M. Kamchatnov, Y. H. Kuo, T. C. Lin, T. L. Horng, S. C. Gou, R. Clift, G. A. El, and R. H. J. Grimshaw, Undular bore theory for the Gardner equation, Phys. Rev. E 86, 036605 (2012). 